\title{
One-pot multi-component green synthesis of highly substituted piperidines
}

\author{
Ravi Bansal*, Pradeep K. Soni, Jyoti Sharma, Santosh K. Bhardwaj and Anand K. Halve
}

School of Studies in Chemistry, Jiwaji University, Gwalior (M.P.) INDIA- 474011

\begin{tabular}{l}
\hline C H R O N I C L E \\
\hline Article history: \\
Received January 2, 2017 \\
Received in revised form \\
March 1, 2017 \\
Accepted March 6, 2017 \\
Available online \\
March 6, 2017 \\
\hline Keywords: \\
Heterocycles \\
Piperidines \\
Multi-component synthesis \\
Catalyst \\
Sodiumlaurylsulfate (SLS) \\
\hline
\end{tabular}

\begin{tabular}{l} 
A B S T R A C T \\
\hline An effective and expeditious method of the synthesis of a highly functionalized piperidines, \\
catalyzed by nontoxic, recyclable and environment friendly sodium lauryl sulfate (SLS), via \\
one-pot multi-component condensation of aldehydes, amines and $\beta$-ketoesters in water at room \\
temperature, has been developed. This new protocol has advantages such as moderate to high \\
yields of products obtained after simple post reaction workup. Structure of the synthesized \\
compounds $4 \mathbf{4}-\mathbf{4 j}$ have been elucidated based on the ${ }^{1} \mathrm{H}$ NMR, ${ }^{13} \mathrm{C}$ NMR, FT-IR spectroscopy \\
and elemental analysis. \\
\end{tabular}

\section{Introduction}

Heterocyclic compounds containing nitrogen atom are wide spread in nature and have significant practical importance because of their applications in medicine and agriculture. ${ }^{1,2}$ They are also used as functional materials. The development of new and efficient methods for the synthesis of $N$-heterocycles is one of the greater interests of modern synthetic organic chemistry. ${ }^{3-5}$

Synthesis of complex heterocyclic molecules can be easily achieved starting from readily available starting materials in a single step multi-component reactions (MCRs). ${ }^{6}$ In most of the cases these reactions are advantageous over the linear step-wise syntheses because of the operational simplicity, shorter reaction time, ecological concerns, low processing costs and avoidance of protection and deprotection processes. ${ }^{7}$

Substituted piperidines are widely present in naturally occurring and synthetic drugs. ${ }^{8}$ A variety of structural features are exhibited by synthetically prepared piperidines including many significant biological activities. Many methods have been extensively studied for the synthesis of piperidines because of their antihistamic, ${ }^{9}$ anti-HIV, ${ }^{10}$ anticancer, ${ }^{11}$ antimicrobial, ${ }^{12}$ anti-malarial, ${ }^{13}$ antiinflammatory, ${ }^{14}$ insecticidal $^{15}$ and other biological activities.

* Corresponding author.

E-mail address: ravibansa1880@gmail.com (R. Bansal)

(C) 2017 Growing Science Ltd. All rights reserved.

doi: $10.5267 /$ j.ccl.2017.3.001 
Recently, many MCRs have been reported for the syntheses of piperidine derivatives in the presence of L-proline/tetrahydrofuran (THF), ${ }^{13}$ indiumtrichloride $\left(\mathrm{InCl}_{3}\right),{ }^{16}$ bromodimethyl sulfoniumbromide (BDMS), ${ }^{17}$ tetrabutylammoniumtribromide (TBATB), ${ }^{18}$ iodine, ${ }^{19}$ cerium ammoniumnitrate $(\mathrm{CAN}),{ }^{20} \quad \mathrm{ZrOCl}_{2} \cdot 8 \mathrm{H}_{2} \mathrm{O},{ }^{21} \quad$ citric acid, ${ }^{22} \quad$ calix $[n]$ arenes, ${ }^{23}$ tris(pentafluorophenyl)borane $\left[\mathrm{B}\left(\mathrm{C}_{6} \mathrm{~F}_{5}\right)_{3}\right],{ }^{24}$ sulfamic acid ${ }^{25}$ and 2,6-pyridinedicarboxylic acid ${ }^{26}$ used as catalysts. Some of these methods are having such draw backs as long reaction times, unsatisfactory yields or use of expensive catalysts. All these prompted us to develop a new simple and greener method of the synthesis of piperidines.

In the present communication we have reported a simple and efficient procedure of one-pot multicomponent synthesis of highly substituted piperidines by the reaction between aromatic aldehydes, anilines and $\beta$-ketoesters in the presence of SLS, used as a catalyst, under mild reaction conditions at room temperature (Scheme 1). SLS is cheap, readily available, versatile, environment friendly and recyclable. The reactions have been carried out in water, what eliminated the use of organic solvents.

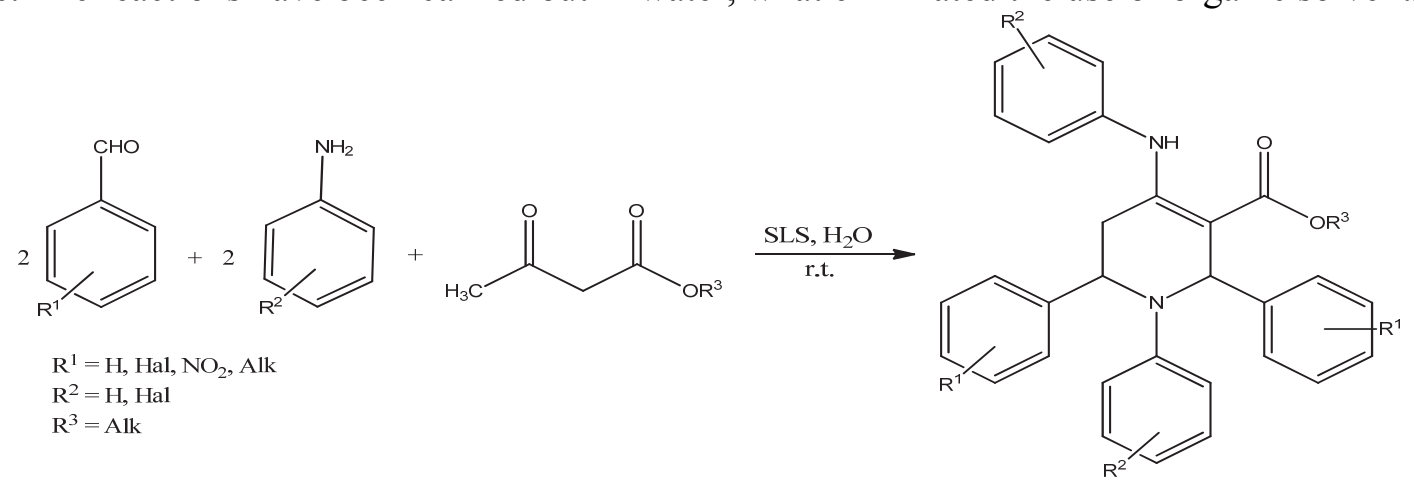

Scheme 1. One-pot multi-componet synthesis of substituted piperidines

\section{Results and Discussion}

Initially benzaldehyde $(2 \mathrm{mmol})$ was treated with aniline $(2 \mathrm{mmol})$ and ethyl acetoacetate $(1$ $\mathrm{mmol}$ ) with water in absence of catalyst. No product was obtained at room temperature after $24 \mathrm{~h}$ (Table 1 , entry 8). To determine the best experimental conditions, the reaction was carried out in the presence of $0.02 \mathrm{~g} \mathrm{SLS}$ in water at $100{ }^{\circ} \mathrm{C}$. The reaction proceeded smoothly to give the corresponding functionalized piperidine in $30 \%$ yield after $24 \mathrm{~h}$ (Table 1, entry 13). When the same reaction was carried out under solvent-free conditions, the product was obtained $25 \%$ yield after $24 \mathrm{~h}$ (Table 1, entry 14). The best results were obtained in the presence of $0.02 \mathrm{~g}$ SLS in water at room temperature (Table 1 , entry 9).

Table 1. Condensation of benzaldehyde, aniline and ethylacetoacetate in different conditions.

\begin{tabular}{cccccc}
\hline Entry & Catalyst & Solvent & Time, h & $\mathbf{T},{ }^{0} \mathbf{C}$ & Yield, $\%$ \\
\hline 1 & $\mathrm{NiCl}_{2}$ & Water & 24 & 50 & 40 \\
2 & $\mathrm{ZnO}_{3}$ & Ethanol & 24 & 80 & No product \\
3 & $\mathrm{Fe}_{2} \mathrm{O}_{3}$ & Ethanol & 24 & 70 & No product \\
4 & $\mathrm{CaO}$ & Ethanol & 8 & 80 & No product \\
5 & $\mathrm{~L}-\mathrm{Proline}$ & Ethanol & 10 & 80 & 40 \\
6 & $\mathrm{CuO}$ & Ethanol & 14 & 60 & No product \\
7 & $\mathrm{Al}_{2} \mathrm{O}_{3}$ & Ethanol & 10 & 50 & No product \\
8 & Without catalyst & Water & 24 & r.t. & No product \\
9 & $\mathrm{SLS}$ & Water & 6 & r.t. & No product \\
10 & Twine-20 & Water & 10 & 50 & No product \\
11 & $\mathrm{Cetrimide}$ & Water & 10 & r.t. & No product \\
12 & Triton X-100 & Water & 10 & 100 & 30 \\
13 & $\mathrm{SLS}$ & Water & 24 & r.t. & 25 \\
\hline
\end{tabular}

Conditions: benzaldehyde $(2 \mathrm{mmol})$, aniline $(2 \mathrm{mmol})$, ethyl acetoacetate $(1 \mathrm{mmol})$, solvent $(10 \mathrm{~mL})$, catalyst $(0.02 \mathrm{~g})$. 
Several substituted benzaldehydes, anilines, methyl/ethyl acetoacetates (EAA) were examined under the optimized reaction conditions. Benzaldehydes with EWG (electron withdrawing) groups underwent the reaction with anilines efficiently to give the corresponding piperidines in moderate to high yields. Aldehydes possessing the EDG groups e.g. $-\mathrm{CH}_{3}$ were less reactive (Table 2, entry 9).

Table 2. Synthesis of substituted piperidines.

\begin{tabular}{cccccccc}
\hline Entry & $\mathbf{R}^{\mathbf{1}}$ & $\mathbf{R}^{\mathbf{2}}$ & $\mathbf{R}^{\mathbf{3}}$ & Product & Time, $\mathbf{h}$ & Yield, $\mathbf{\%}$ & M.p., $\mathbf{C}^{\mathbf{0}}$ \\
\hline 1 & $\mathrm{H}$ & $\mathrm{H}$ & $\mathrm{Et}$ & $\mathbf{4 a}$ & 6 & 90 & 174 \\
2 & $\mathrm{H}$ & $4-\mathrm{Cl}$ & $\mathrm{Et}$ & $\mathbf{4 b}$ & 6 & 85 & 220 \\
3 & $2-\mathrm{F}$ & $\mathrm{H}$ & $\mathrm{Et}$ & $\mathbf{4 c}$ & 7 & 65 & 128 \\
4 & $4-\mathrm{Cl}$ & $\mathrm{H}$ & $\mathrm{Et}$ & $\mathbf{4 d}$ & 6 & 95 & 215 \\
5 & $4-\mathrm{NO}_{2}$ & $\mathrm{H}$ & $\mathrm{Me}$ & $\mathbf{4 e}$ & 6 & 80 & 236 \\
6 & $3-\mathrm{NO}_{2}$ & $\mathrm{H}$ & $\mathrm{Et}$ & $\mathbf{4 f}$ & 7 & 80 & 247 \\
7 & $4-\mathrm{F}$ & $\mathrm{H}$ & $\mathrm{Et}$ & $\mathbf{4 g}$ & 6 & 80 & 170 \\
8 & $\mathrm{H}$ & $4-\mathrm{F}$ & $\mathrm{Et}$ & $\mathbf{4 h}$ & 7 & 80 & 144 \\
9 & $4-\mathrm{Me}$ & $4-\mathrm{Cl}$ & $\mathrm{Et}$ & $\mathbf{4 L}$ & 8 & 75 & 234 \\
10 & $4-\mathrm{OH}$ & $\mathrm{H}$ & $\mathrm{Et}$ & $\mathbf{4 j}$ & 6 & 90 & 233 \\
\hline
\end{tabular}

Conditions: aromatic aldehyde $(2 \mathrm{mmol})$ aromatic amine $(2 \mathrm{mmol}), \beta$-ketoester $(1 \mathrm{mmol})$, SLS $(0.02 \mathrm{~g})$, water $(10 \mathrm{~mL})$, room temperatue.

The structures of newly synthesized compounds $\mathbf{4 a - 4} \mathbf{j}$ has been confirmed based on the $1 \mathrm{H}$ NMR, ${ }^{13} \mathrm{C}$ NMR and FT-IR spectroscopic and elemental analysis data.

The temperature seems does not have any significant effect on the products yield. The yields of the products did not also improved when the amount of SLS was increased. The results are presented in Table 3.

Table 3. Effect of SLS loading on the synthesis of piperidine $\mathbf{4 a}$ at room temperature.

\begin{tabular}{cccc}
\hline Entry & SLS,, $\mathbf{g}$ & Time, $\mathbf{h}$ & Yield, $\%$ \\
\hline 1 & 0.005 & 6 & 20 \\
2 & 0.01 & 6 & 30 \\
3 & 0.02 & 6 & 95 \\
4 & 0.05 & 6 & 30 \\
5 & 0.10 & 6 & No product \\
\hline
\end{tabular}

Conditions: benzaldehyde $(2 \mathrm{mmol})$, aniline $(2 \mathrm{mmol})$, ethyl acetoacetate $(1 \mathrm{mmol})$, SLS $(0.02 \mathrm{~g})$, water $(10 \mathrm{~mL})$, room temperature.

Based on previous literature records, ${ }^{13}, 16-23$ it is reasonable to assume following mechanism of the reaction. Piperidines 4 results from initial condensation of aromatic aldehydes (1) and $\beta$-ketoesters (3) with anilines (2), in the presence of SLS, to give enamine 5 and imine $\mathbf{6}$ (Scheme 2) which undergone intermolecular Mannich-type reaction to produce intermediate 7. The reaction between intermediate 7 and $\mathbf{1}$ gives intermediate $\mathbf{8}$ by the elimination of $\mathrm{H}_{2} \mathrm{O}$. Tautomerization of $\mathbf{8}$ generates intermediate $\mathbf{9}$, which immediately undergoes intra-molecular Mannich-type reaction to give intermediate 10. Finally, the 10 tautomerizes to generate the desired piperidines derivative 4 owing to conjugation with the ester group.

This reaction can be regarded as an efficient approach for the preparation of synthetically and pharmaceuptically important piperidine systems.

\section{Conclusions}

A general methodology of the formation of highly functionalized piperidines from commonly available starting materials, in presence of catalytic amounts of sodiumlaurylsulfate, via one-pot three component reaction is reported. The salient features of this protocol are good yields, mild reaction conditions, environment friendly, superior atom economy and the readily accessibility of the catalyst. 
In addition, we proposed the possibility for the formation of piperidines via double Mannich-type intermediates.

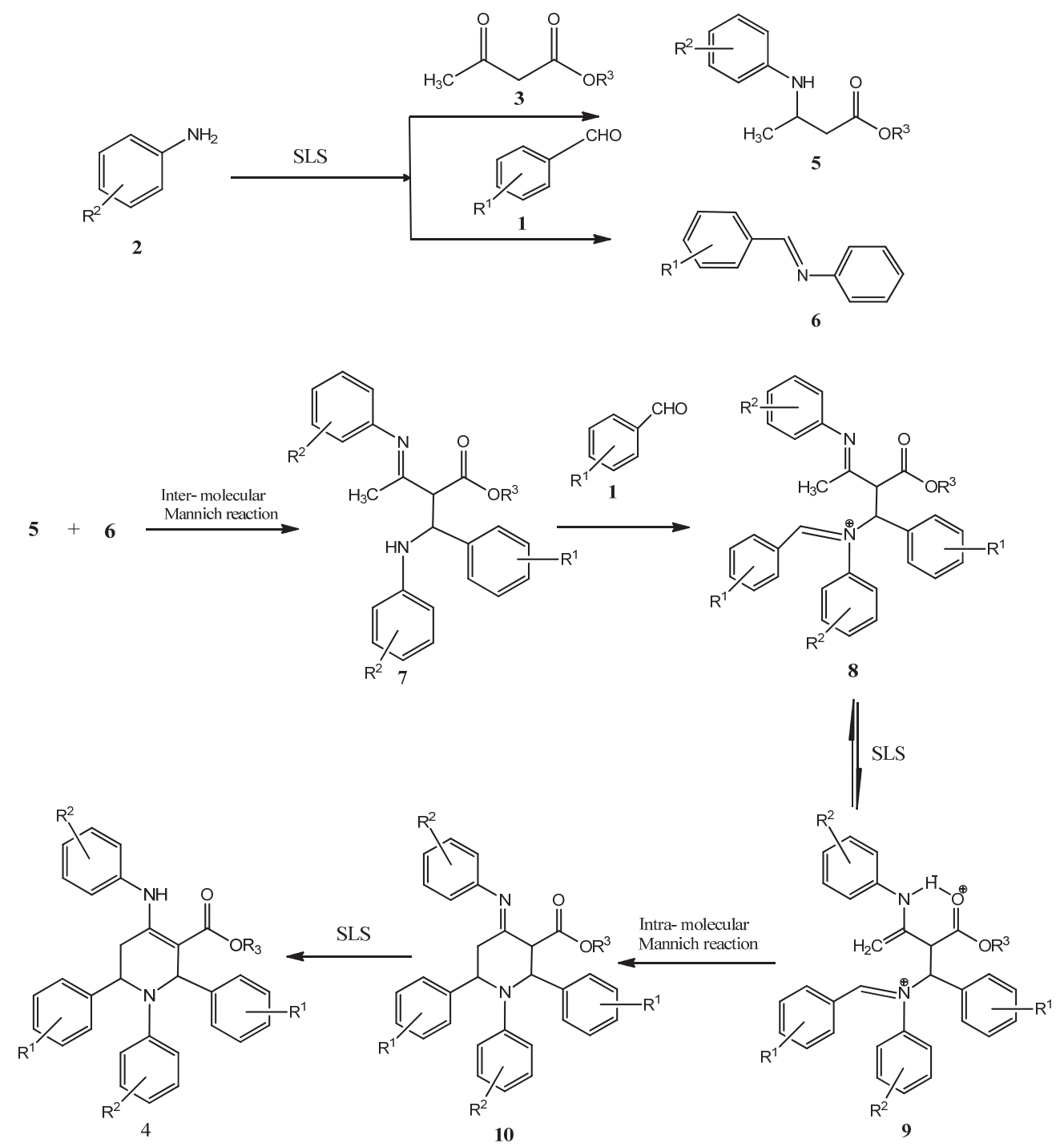

Scheme 2. Proposed molecular mechanism

\section{Acknowledgement}

Authors gratefully acknowledge to Defence Research Development Establishment Gwalior (M.P.) for ${ }^{1} \mathrm{H}$ NMR, ${ }^{13} \mathrm{C}$ NMR and FT-IR spectra. Authors also wish to acknowledge the help rendered by the Head, School of Studies in Chemistry, Jiwaji University, Gwalior (M.P.).

\section{Experimental}

\subsection{Materials and Methods}

All the chemicals were received commercially and used without further purification. Melting points were determined in melting point apparatus, using open capillary tube and are uncorrected. NMR spectra were recorded with a Bruker AV III spectrometer at $400 \mathrm{MHz}\left({ }^{1} \mathrm{H}\right.$ NMR $)$ and $100 \mathrm{MHz}\left({ }^{13} \mathrm{C}\right.$ $\mathrm{NMR}$ ) using $\mathrm{CDCl}_{3}$ as the solvent with tetramethylsilane (TMS) as internal standard. FT-IR spectra of 
compounds were recorded using $\mathrm{KBr}$ pellets on Shimadzu IR Affinity-1, Fourier-Transform infrared spectrometer.

\subsection{General procedure}

A mixture of aromatic amine $2(2 \mathrm{mmol})$ and $\beta$-ketoester $3(1 \mathrm{mmol})$ in $10 \mathrm{ml}$ water was stirred for $20 \mathrm{~min}$ in the presence of $0.02 \mathrm{~g}$ sodiumlaurylsulfate at room temperature. Next the aromatic aldehyde $1(2 \mathrm{mmol})$ was added and the reaction mixture was stirred for the time indicated in Table 2 . The progress of reactions was monitored by thin layer chromatography (TLC), eluted with ethyl acetate and n-hexane (3:7) mixture. After completion of the reaction, a thick precipitate was filtered off and washed with water. The crystalline pure products were obtained by further recrystalization from ethanol.

\subsection{Physical and Spectral Data}

Ethyl-1,2,6-triphenyl-4-(phenylamino)-1,2,5,6-tetrahydropyridine-3-carboxylate (4a) Yield $=90$, White yellow solid, melting point $=174{ }^{\circ} \mathrm{C}$, Elemental Analysis Data found (required \%) $\mathrm{C}=80.85$ (80.98), $\mathrm{H}=6.30$ (6.37), $\mathrm{N}=5.80$ (5.90), $\mathrm{O}=6.68$ (6.74). FT-IR (KBr): $3244.27(\mathrm{~N}-\mathrm{H})$, $1651.07(\mathrm{C}=\mathrm{O}), 1581.63(\mathrm{C}=\mathrm{C}) \mathrm{cm}^{-1} .{ }^{1} \mathbf{H}$ NMR $\left(400 \mathrm{MHz}, \mathrm{CDCl}_{3}\right) \mathrm{ppm}: 1.19(\mathrm{t}, J=7.2,6.8 \mathrm{~Hz}, 3 \mathrm{H})$, $1.41(\mathrm{dd}, J=2,15.2 \mathrm{~Hz}, 1 \mathrm{H}), 2.79(\mathrm{dd}, J=6,5.6,15 \mathrm{~Hz}, 1 \mathrm{H}), 4.09-4.26(\mathrm{~m}, 1 \mathrm{H}), 4.28-4.38(\mathrm{~m}, 1 \mathrm{H})$, $5.07(\mathrm{~d}, J=4 \mathrm{~Hz}, 1 \mathrm{H}), 6.12-6.21(\mathrm{~m}, 2 \mathrm{H}), 6.99(\mathrm{~s}, 1 \mathrm{H}), 7 .(\mathrm{d}, J=8.4 \mathrm{~Hz}, 2 \mathrm{H}), 7.02(\mathrm{t}, J=7.2 \mathrm{~Hz}, 1 \mathrm{H})$, 7.05-7.10 (m, 5H), 7.12-7.18 (m, 2H), 7.20-7.25 (m, 6H), $7.58(\mathrm{~d}, J=7.6 \mathrm{~Hz}, 2 \mathrm{H}), 10.21(\mathrm{~s}, 1 \mathrm{H}) .{ }^{13} \mathbf{C}$ NMR (100 MHz, $\left.\mathrm{CDCl}_{3}\right)$ ppm: 14.9, 33.7, 55.2, 58.3, 59.8, 98.3, 113.0, 116.2, 125.8, 125.9, 126.4, $126.5,126.7,127.2,128.3,128.7,128.9,128.9,137.9,142.9,144.1,147.0,156.2,168.3$.

Ethyl-1-(4-chlorophenyl)-4(4chlorophenyl)amino),2,6-diphenyl-1,2,5,6-tertahydropyridine-3carboxylate (4b)

Yield $=85$, Yellow solid, melting point $=220{ }^{\circ} \mathrm{C}$, Elemental Analysis Data found (required \%) $\mathrm{C}=$ 70.62 (70.72), $\mathrm{H}=5.10$ (5.19), $\mathrm{Cl}=12.95$ (13.05), $\mathrm{N}=5.10$ (5.15), $\mathrm{O}=5.80$ (5.89). FT-IR (KBr): $3246.20(\mathrm{~N}-\mathrm{H}), 1645.28(\mathrm{C}=\mathrm{O}), 1492.90(\mathrm{C}=\mathrm{C}) \mathrm{cm}^{-1} .{ }^{1} \mathbf{H}$ NMR $\left(400 \mathrm{MHz}, \mathrm{CDCl}_{3}\right) \mathrm{ppm}$ : 1.52-1.49 (t, $J=6.0 \mathrm{~Hz}, 3 \mathrm{H}), 2.75-2.72(\mathrm{~d}, J=12.0 \mathrm{~Hz}, 1 \mathrm{H}), 2.91-2.87(\mathrm{dd}, J=24.0,4.0 \mathrm{~Hz}, 1 \mathrm{H}), 4.39-4.35(\mathrm{~m}$, $1 \mathrm{H}), 4.52-4.49(\mathrm{~m}, 1 \mathrm{H}), 5.14-5.13(\mathrm{~s}, 1 \mathrm{H}), 6.21-6.19(\mathrm{~d}, J=8.0 \mathrm{~Hz}, 2 \mathrm{H}), 6.43(\mathrm{~s}, 1 \mathrm{H}), 6.48-6.46(\mathrm{~d}, J$ $=8.0 \mathrm{~Hz}, 2 \mathrm{H}), 7.04-7.02(\mathrm{~d}, J=8.0 \mathrm{~Hz}, 2 \mathrm{H}), 7.09-7.07(\mathrm{~d}, J=8.0 \mathrm{~Hz}, 2 \mathrm{H}), 7.19-7.17(\mathrm{~d}, J=8.0 \mathrm{~Hz}$, 2H), 7.31-7.27 (m, 8H), 10.26 (br s, $1 \mathrm{H}) .{ }^{13} \mathbf{C}$ NMR (100 MHz, $\left.\mathrm{CDCl}_{3}\right)$ ppm: 14.8, 33.5, 55.3, 58.3, $59.9,98.7,114.0,121.2,126.3,126.5,126.6,127.0,127.5,128.4,128.7,128.8,129.0,131.4,136.4$, $142.3,143.3,145.5,155.4,168.1$.

\section{Ethyl-2,6-bis(4-chlorophenyl)-1,2,5,6-tetrahydro-1-phenyl-4-(phenylamino)pyridine-3- carboxylate (4d)}

Yield $=95$, White solid, melting point $=215^{\circ} \mathrm{C}$, Elemental Analysis Data found (required \%) $\mathrm{C}=$ 70.65 (70.72), $\mathrm{H}=5.15$ (5.19), $\mathrm{Cl}=12.95$ (13.05), $\mathrm{N}=5.08$ (5.15), $\mathrm{O}=5.80$ (5.89). FT-IR (KBr): $3057.17(\mathrm{~N}-\mathrm{H}), 1649.14(\mathrm{C}=\mathrm{O}), 1485(\mathrm{C}=\mathrm{C}) \mathrm{cm}^{-1} .{ }^{1} \mathbf{H}$ NMR $\left(400 \mathrm{MHz}, \mathrm{CDCl}_{3}\right)$ ppm: 1.47-1.43 (t, $J=$ $12.0 \mathrm{~Hz}, 3 \mathrm{H}), 2.76-2.72(\mathrm{~d}, J=16.0 \mathrm{~Hz}, 1 \mathrm{H}), 2.85-2.80(\mathrm{dd}, J=32.0,4.0 \mathrm{~Hz}, 1 \mathrm{H}), 4.36-4.28(\mathrm{~m}, 1 \mathrm{H})$, 4.48-4.40 (m, 1H), $5.09(\mathrm{~s}, 1 \mathrm{H}), 6.36(\mathrm{~s}, 1 \mathrm{H}), 6.41-6.39(\mathrm{~d}, J=8.0 \mathrm{~Hz}, 2 \mathrm{H}), 6.46-6.44(\mathrm{~d}, J=8.0 \mathrm{~Hz}$, $2 \mathrm{H}), 6.66-6.62(\mathrm{t}, J=8.0 \mathrm{~Hz}, 1 \mathrm{H}), 7.17-7,04(\mathrm{~m}, 7 \mathrm{H}), 7.27-7.22(\mathrm{~m}, 6 \mathrm{H}), 10.31(\mathrm{br} \mathrm{s}, 1 \mathrm{H}) .{ }^{13} \mathbf{C}$ NMR (100 MHz, $\mathrm{CDCl}_{3}$ ) ppm: 14.8, 33.7, 54.7, 57.4, 59.9, 97.8, 112.9, 116.7, 125.7, 125.9, 127.8, 128.0, $128.4,128.8,129.0,129.1,132.1,132.9,137.7,140.9,142.5,146.5,155.8,167.9$. 
Methyl-1,2,5,6-tetrahydro-2,6-bis(4-nitrophenyl)-1-phenyl-4-(phenylamino)pyridine-3carboxylate (4e)

Yield $=80$, Yellow solid, melting point $=236{ }^{\circ} \mathrm{C}$, Elemental Analysis Data found (required \%) $\mathrm{C}=$ 67.58 (67.63), $\mathrm{H}=4.70$ (4.76), $\mathrm{N}=10.10$ (10.18), $\mathrm{O}=17.40$ (17.44). FT-IR (KBr): 3239 (N-H), 1667 $(\mathrm{C}=\mathrm{O}), 1530(\mathrm{C}=\mathrm{C}) \mathrm{cm}^{-1} .{ }^{1} \mathbf{H}$ NMR $\left(400 \mathrm{MHz}, \mathrm{CDCl}_{3}\right) \mathrm{ppm}: 2.87(\mathrm{~s}, 2 \mathrm{H}), 3.97(\mathrm{~s}, 3 \mathrm{H}), 5.27(\mathrm{~s}, 1 \mathrm{H})$, 6.44-6.39 (t, $J=10.0 \mathrm{~Hz}, 3 \mathrm{H}), 6.48(\mathrm{~s}, 1 \mathrm{H}), 6.71-6.68(\mathrm{t}, J=6.0 \mathrm{~Hz}, 1 \mathrm{H}), 7.13-7.04(\mathrm{t}, J=18.0 \mathrm{~Hz}$, $2 \mathrm{H}), 7.16(\mathrm{~s}, 3 \mathrm{H}), 7.30-7.26(\mathrm{~m}, 2 \mathrm{H}), 7.51-7.49(\mathrm{~d}, J=8.0 \mathrm{~Hz}, 2 \mathrm{H}), 8.17-8.12(\mathrm{t}, J=10.0 \mathrm{~Hz}, 5 \mathrm{H})$, 10.28 (br s, $1 \mathrm{H}, \mathrm{NH}) .{ }^{13} \mathbf{C}$ NMR $\left(100 \mathrm{MHz}, \mathrm{CDCl}_{3}\right.$ ) ppm: 25.6, 33.6, 51.5, 55.2, 57.3, 67.9, 96.7, 112.9, $117.7,123.7,123.8,123.9,124.0,125.5,126.5,127.4,127.4,127.6,127.8,129.1,129.2$, 129.4, 137.1, $145.8,146.8,147.3,149.8,151.6,155.5,167.9$.

\section{Ethyl-1,2,5,6-tetrahydro-2,6-bis(3-nitrophenyl)-1-phenyl-4-(phenylamino)pyridine-3- carboxylate (4f)}

Yield $=80$, Yellow solid, melting point $=247{ }^{\circ} \mathrm{C}$, Elemental Analysis Data found (required \%) $\mathrm{C}=$ 68.00 (68.07), $\mathrm{H}=4.95$ (5.00), $\mathrm{N}=9.87$ (9.92), $\mathrm{O}=16.94$ (17.00). FT-IR (KBr): $3233(\mathrm{~N}-\mathrm{H}), 1659$ $(\mathrm{C}=\mathrm{O}), 1504(\mathrm{C}=\mathrm{C}) \mathrm{cm}^{-1} .{ }^{1} \mathbf{H}$ NMR $\left(400 \mathrm{MHz}, \mathrm{CDCl}_{3}\right) \mathrm{ppm}: 1.57-1.54(\mathrm{t}, J=6.0 \mathrm{~Hz}, 3 \mathrm{H}), 2.92-2.92$ $(\mathrm{s}, 2 \mathrm{H}), 4.41-4.37(\mathrm{~m}, 1 \mathrm{H}), 4.59-4.55(\mathrm{~m}, 1 \mathrm{H}), 5.36(\mathrm{~s}, 1 \mathrm{H}), 6.44-6.42(\mathrm{~m}, 2 \mathrm{H}), 6.48-6.46(\mathrm{~d}, J=8.0$ $\mathrm{Hz}, 2 \mathrm{H}), 6.52(\mathrm{~s}, 1 \mathrm{H}), 6.74-6.71(\mathrm{t}, J=6.0 \mathrm{~Hz}, 1 \mathrm{H}), 7.19-7.11(\mathrm{~m}, 5 \mathrm{H}), 7.51-7.46(\mathrm{~m}, 3 \mathrm{H}), 7.68-7.66$ $(\mathrm{d}, J=8.0 \mathrm{~Hz}, 1 \mathrm{H}), 7.98(\mathrm{~s}, 1 \mathrm{H}), 8.17-8.12(\mathrm{~m}, 2 \mathrm{H}), 8.35(\mathrm{~s}, 1 \mathrm{H}), 10.38(\mathrm{br} \mathrm{s}, 1 \mathrm{H}) .{ }^{13} \mathbf{C} \mathbf{N M R}(100$ $\mathrm{MHz}, \mathrm{CDCl}_{3}$ ) ppm: 14.8, 25.6, 33.8, 55.2, 57.0, 60.3, 67.9, 97.0, 113.1, 117.7, 121.4,121.7, 121.8, $122.5,125.6,126.5,129.1,129.3,129.4,129.7,132.3,132.6,137.2,144.5,145.8,146.4,148.6,148.7$, $155.3,167.7$.

\section{References}

1. Lichtenthaler F. W. (2002) Unsaturated $O$ - and $N$-heterocycles from carbohydrate feedstocks. Acc. Chem. Res., 35 (9) 728-737.

2. Litvinov V. P. (2003) Multi-component cascade heterocyclisation as a promising route to targeted synthesis of polyfunctional pyridines. Russ. Chem. Rev., 72 (1) 69-85.

3. Padwa A., and Waterson A. G. (2000) Synthesis of nitrogen heterocycles using the intramolecular pummerer reaction. Curr. Org. Chem., 4 (2) 175-203.

4. Orru R. V. A. and de Greef M. (2003) Recent advances in solution-phase multi-component methodology for the synthesis of heterocyclic compounds. Synthesis., 2003 (10) 1471-1499.

5. Kirsch G., Hesse S., and Comel A. (2004) Synthesis of five and six membered heterocycles through palladium-catalyzed reaction. Curr. Org. Synth., 1 (1) 47-63.

6. (a) Frederic L. M., Constantieux T., and Rodriguez J. (2005) Multi-component domino reaction from $\beta$-ketoamides: highly efficient access to original polyfunctionalized 2,6-diazabi cyclo[2.2.2]octane cores. J. Am. Chem. Soc., 127 (49) 17176-17177.

(b) Simon C., Peyronel J. F., and Rodriguez J. (2001) A new multi-component domino reaction of 1,3-dicarbonyl compounds: one-pot access to polycyclic $N / O-, N / S$-, and $N / N$-aminals. $J$. Org. Lett., 3 (14) 2145-2148.

7. (a) Majumdar K. C., Ponra S., and Ghosh T. (2012) Green approach to highly functionalized thiopyrano derivatives via domino multi-component reaction in water. RSC Adv., 2 (3) 1144 1152 .

(b) Kumaravel K., and Vasuki G. (2009) Four-component catalyst-free reaction in water: Combinatorial library synthesis of novel 2-amino-4-(5-hydroxy-3-methyl-1H-pyrazol-4-yl)4H-chromene-3-carbonitrile derivatives. Green Chem., 11 (12) 1945-1947.

(c) Girling P. R., Batsanov A. S., Shen H. C., and Whiting A. (2012) A multi-component formal $[1+2+1+2]$ cycloaddition for the synthesis of dihydropyridines. Chem. Commun., 48 (40) 4893-4895. 
(d) Hardy S., and Martin S. F. (2011) Multi-component assembly and diversification of novel heterocyclic scaffolds derived from 2-arylpiperidines. Org. Lett., 13 (12) 3102-3105.

8. (a) Tawara J. N., Lorenz P., and Stermitz F. R. (1999) 4-Hydroxylated piperidines and nmethyleuphococcinine (1-methyl-3-granatanone) from picea (spruce) species. identification and synthesis. J. Nat. Prod., 62 (2) 321-323.

(b) Lebold T. B., Leduc A. B., and Kerr M. A. (2009) Zn(II)-catalyzed synthesis of piperidines from propargyl amines and cyclopropane. Org. Lett., 11 (16) 3770-3772.

(c) Watson P. S., Jiang B., and Scott B. (2000) A diastereoselective synthesis of 2,4disubstituted piperidines: scaffolds for drug discovery. Org. Lett., 2 (23) 3679-3681.

9. Saxena M., Gaur S., Prathipati P., and Saxena A. K. (2006) Synthesis of some substituted pyrazinopyridoindoles and 3D QSAR studies along with related compounds: piperazines, piperidines, pyrazinoisoquinolines, and diphenhydramine, and its semi-rigid analogs as antihistamines $\left(\mathrm{H}_{1}\right)$. Bioorg. Med. Chem., 14 (24) 8249-8258.

10. Imamura S., Nishikawa Y., Ichikawa T., Hattori T., Matsushita Y., Hashiguchi S., Kanzaki N., Iizawa Y., Baba M., and Sugihara Y. (2005) CCR5 antagonists as anti-HIV-1 agents. Part 3: Synthesis and biological evaluation of piperidine-4-carboxamide derivatives. Bioorg. Med. Chem., 13 (2) 397-416.

11. Srinivas C., Kumar C. N. S. P., Raju B. C., Rao V. J., Naidu V. G. M., Ramakrishn S., and Diwan P. V. (2009) First stereoselective total synthesis and anticancer activity of new amide alkaloids of roots of pepper. Bioorg. Med. Chem. Lett., 19 (20) 5915-5918.

12. Umamatheswari S., Balaji B., Ramanathan M., and Kabilan S. (2010) Synthesis, antimicrobial evaluation and QSAR studies of novel piperidin-4-yl-5-spiro-thiadiazoline derivatives. Bioorg. Med. Chem. Lett., 20 (23) 6909-6914.

13. Misra M., Pandey S. K., Pandey V. P., Pandey J., Tripathi R., and Tripathi R. P. (2009) Organocatalyzed highly atom economic one-pot synthesis of tetrahydropyridines as antimalarials. Bioorg. Med. Chem., 17 (2) 625-633.

14. Khanum S. A., Girish V., Suparshwa S. S., and Khanum N. F. (2009) Benzophenone-N-ethyl piperidine ether analogues. Synthesis and efficacy as anti-inflammatory agent. Bioorg. Med. Chem. Lett., 19 (7) 1887-1891.

15. Bandaraa K. A. N. P., Kumara V., Jacobsson U., and Molleyresc L. P. (2000) Insecticidal piperidine alkaloid from microcospaniculata stem bark. Phytochem., 54 (1) 29-32.

16. Clark P. A., Zaytzev A. V., and Whitwood A. C. (2007) Pot, atom and step economic (PASE) synthesis of highly functionalized piperidines: a five-component condensation. Tetrahedron Lett., 48 (30) 5209-5212.

17. Khan A. T., Parvin T., and Choudhury L. H. (2008) Effects of substituents in the $\beta$-position of 1,3-dicarbonyl compounds in bromodimethylsulfonium bromide-catalyzed multi-component reactions: a facile access to functionalized piperidines. J. Org. Chem., 73 (21) 8398-8402.

18. Khan A. T., Lal M., Khan M. M., and Bannuru K. K. R. (2010) Synthesis of highly functionalized piperidines by one-pot multi-component reaction using tetrabutylammoniumtribromide (TBATB). Tetrahedron Lett., 51 (48) 4419-4424.

19. Khan A. T., Khan M. M., and Bannuru K. K. R. (2010) Iodine catalyzed one-pot fivecomponent reactions for direct synthesis of densely functionalized piperidines. Tetrahedron., 66 (39) 7762-7772.

20. Wang H. J., Mo L. P., and Zhang Z. H. (2011) Cerium ammonium nitrate-catalyzed multicomponent reaction for efficient synthesis of functionalized tetrahydropyridines. ACS. Comb. Sci., 13 (2) 181-185.

21. Mishra S., and Ghosh R. (2011) Efficient one-pot synthesis of functionalized piperidine scaffolds via $\mathrm{ZrOCl}_{2} .8 \mathrm{H}_{2} \mathrm{O}$ catalyzed tandem reactions of aromatic aldehydes with amines and acetoacetic esters. Tetrahedron Lett., 52 (22) 2857-2861.

22. Madanifar Z., Maghsoodlou M., Kangani M., and Hazeri N. (2015) Citric acid, a green catalyst for the one-pot, multi-component synthesis of highly substituted piperidines. Res. Chem. Intermed., 41 (12) 9863-9869. 
23. Palermo V., Sathicq A., Liberto N., Fernandes S., Langer P., Jios J., and Romanelli G. (2016) Calix[n]arenes: active organocatalysts for the synthesis of densely functionalized piperidines by one-pot multi-component procedure. Tetrahedron Lett., 57 (19) 2049-2054.

24. Prajapati S. K., Nagarsenkar A., Guggilapu S. D., and Babu B. N. (2015) B $\left(\mathrm{C}_{6} \mathrm{~F}_{5}\right)_{3}$ as versatile catalyst: an efficient and mild protocol for the one-pot synthesis of functionalized piperidines and 2-substituted benzimidazole derivatives. Tetrahedron Lett., 56 (48) 6795-6799.

25. Patil D., Chandam D., Mulik A., Patil P., Jagdale S., and Deshmukh M. (2015) Multicomponent synthesis of highly functionalized piperidines using sulfamic acid as a heterogeneous and cost effective catalyst. Indian J. Chem., 154 (B) (04) 545-550.

26. Khan M. M., Khan S., Iqbal S., Saigal., and Yousuf R. (2016) Synthesis of functionalized dihydro-2-oxypyrroles and tetrahydropyridines using 2,6-pyridinedicarboxylic acid as an efficient and mild organo-catalyst. New J. Chem., 40 (9) 7504-7512.

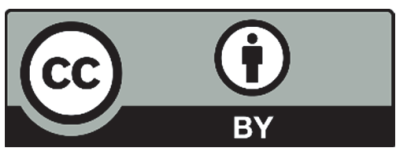

(C) 2016 by the authors; licensee Growing Science, Canada. This is an open access article distributed under the terms and conditions of the Creative Commons Attribution (CC-BY) license (http://creativecommons.org/licenses/by/4.0/). 